\title{
Relação entre Ordem de Nascimento e Estilos Interpessoais
}

\author{
Mauro de Oliveira Magalhães \\ Universidade Federal da Bahia
}

\begin{abstract}
RESUMO
O estilo interpessoal é um aspecto da personalidade referente à forma particular do indivíduo participar e obter influência no meio social. Origina-se na infância a partir das primeiras interações no grupo familiar. Sugere-se que a posição do indivíduo na genitura familiar, denominada ordem de nascimento, é uma variável importante neste processo. Esta pesquisa investigou efeitos combinados de sexo e ordem de nascimento sobre o estilo interpessoal. Uma amostra de 435 estudantes universitários (196 homens e 239 mulheres), com idades entre 18 e 40 anos $(\underline{M}=23,3)$, respondeu ao BASIS-A (Basic Adlerian Scales of Interpersonal Styles) e a um breve questionário demográfico. Foram encontradas interações entre sexo e ordem de nascimento para diferenças de estilo interpessoal. Mulheres caçulas revelaram maior tendência a buscar sucesso e aprovação social do que mulheres primogênitas e homens caçulas. Entre os homens, os caçulas revelaram menor necessidade de aprovação social do que primogênitos e filhos únicos. E primogênitos mostraram maior necessidade de atender às convenções sociais e obter sucesso. A interação entre sexo e ordem de nascimento se mostrou relevante para compreender o desenvolvimento da personalidade no contexto das relações familiares.
\end{abstract}

Palavras-chave: ordem de nascimento; estilos interpessoais; personalidade.

\section{ABSTRACT \\ Relation Between Birth Order and Interpersonal Styles}

Interpersonal style is an aspect of personality related to the particular way individuals participate and gain influence in social contexts. It has its origin in childhood's first social interactions within the family group. It is suggested that the individual position in the family structure, namely birth order, is an important variable in this process. The present study investigated combined effects of sex and birth order on interpersonal style. A sample of 435 college students (196 men and 239 women) with ranging in age from 18 to 40 years $(\underline{M}=23,3)$ answered the BASIS-A (Basic Adlerian Scales of Interpersonal Styles) and a brief demographic questionnaire. Interactions between sex and birth order were found. Lastborn women showed greater tendency to search for success and social approval than firstborn women and lastborn men. Among men, lastborn revealed less need for social approval compared to firstborn and only children. First born men showed a higher need to attend social conventions and obtain success. The interaction between sex and birth order was relevant for the understanding of personality development in the context of family relations.

Keywords: birth order; interpersonal styles; personality.

Desde as ideias de Adler (1954/1912), realizou-se uma quantidade considerável de pesquisas sobre diferenças individuais relacionadas à ordem de nascimento ou posição ordinal no grupo de irmãos (Sulloway, 1999, 2001). A influência da ordem de nascimento sobre a personalidade é uma questão que ocupou mais de 2000 estudos nos últimos 75 anos (Beer \& Horn, 2000). As pesquisas têm revelado que os irmãos criados na mesma família são quase tão diferentes quanto os indivíduos sem laços de paren- tesco (Healey \& Ellis, 2007; Sulloway, 1999). Portanto, uma das questões mais intrigantes para a psicologia da personalidade é descobrir por que os irmãos são tão diferentes. Numa perspectiva darwiniana, Sulloway (1999) defende que a personalidade é o repertório das estratégias que cada indivíduo desenvolve no esforço de sobreviver à infância. As diferenças marcantes entre irmãos podem ser entendidas por um processo de diversificação evolutiva, que torna possível a coexistência de organismos com necessida- 
des ecológicas idênticas num mesmo ambiente. Explica-se a seguir.

Na medida em que as crianças se desenvolvem, cada uma seleciona um nicho no ambiente familiar. $\mathrm{O}$ termo nicho deve ser entendido aqui como um papel e identidade que possibilitem acesso aos recursos sociais e materiais disponíveis no contexto da família. Os primeiros filhos têm maior possibilidade de escolha e, na medida em que o fazem, reduzem as alternativas dos mais jovens, que tendem a escolher nichos ainda desocupados ou a criar opções diferentes. Este é o processo de diversificação evolutiva. Ao desenvolver interesses e habilidades diferentes, os irmãos minimizam a competição direta e continuam lutando pelo investimento parental (Sulloway, 1999). Por outro lado, Dreikurs (1963) acrescenta que "as crianças em nossa sociedade democrática e competitiva, vivem comparativamente; isto é, cada uma se move na direção que a outra falhou, ou encontra dificuldades, e evita atividades nas quais outros obtêm sucesso" (p. 252) Por exemplo, se o primogênito é excelente na escola, os nascidos mais tarde provavelmente não se interessarão tanto pelos estudos, mas sim por outras atividades onde o primogênito possa mostrar falta de interesse ou habilidade. Estes processos de seleção de interesses, papéis e identidades têm sido usados para explicar as diferenças de personalidade apresentadas por irmãos (Adler, 1954/1912; Dreikurs, 1963; Eckstein, 2000; Sulloway, 1999). A posição ocupada por cada criança lhe trará obstáculos particulares e lhe exigirá estratégias e traços que podem vir a se tornar características marcantes do seu comportamento nos próximos estágios de vida.

Ernst e Angst (1983) revisaram 40 anos de pesquisas sobre o tema e concluíram que os efeitos encontrados foram superestimados e apontaram limitações em muitos delineamentos utilizados. Sulloway (1999, 2001) retomou as análises de Ernst e Angst (1983) e selecionou 196 pesquisas que atenderam aos padrões que estes autores consideraram adequados. A análise destes estudos, melhor delineados, revelou que existem resultados recorrentes e confiáveis sobre as relações entre ordem de nascimento e personalidade. Sulloway (1999) concluiu que, "apesar de todas as críticas, a bibliografia sobre a ordem de nascimento exibe tendências comportamentais sistemáticas" (p. 81). O argumento de Sulloway $(1999,2001)$, de que a competição entre irmãos pelo investimento parental leva as crianças a cultivar nichos familiares associados à ordem de nascimento, recebeu apoio empírico de inúmeras pesquisas recentes (Healey \& Ellis, 2007;
Saroglou \& Fiasse, 2003; Zweigenhaft, 2002). E, nas últimas décadas, uma nova proliferação de investigações sobre o tema levou Zajonc e Mullally (1997) a afirmar que "a ordem de nascimento tem sido rapidamente restabelecida como um fator relevante em psicologia" (p. 698).

Sulloway (1999) organizou suas hipóteses sobre a relação entre personalidade e ordem de nascimento de acordo com as cinco grandes dimensões de personalidade (extroversão, conciliatividade, abertura à experiência, escrupulosidade e neuroticismo). Entre os 196 estudos analisados, 72 apresentaram resultados compatíveis com as hipóteses lançadas pelo autor, 110 indiferentes e 14 refutações. Sobre a dimensão de extroversão, as análises indicaram que primogênitos são mais extrovertidos em termos de maior assertividade e disposição para a liderança. No que se refere à sociabilidade, outra faceta da extroversão, os caçulas se destacaram como mais prestativos e populares. Neste sentido, revelaram serem mais aquiescentes e despreocupados, características associadas à dimensão de conciliatividade/amabilidade (do original agreeableness) da taxonomia dos cinco grandes fatores. A abertura à experiência foi negativamente associada à posição do primogênito, pois este tende a ser mais convencional e tradicionalista, com estreita identificação com seus pais. Deste modo, o primogênito acaba por ser mais responsável, voltado para as realizações, organizado e planejador; sendo que estes traços correspondem à dimensão de escrupulosidade. E, por fim, quanto à dimensão neuroticismo, os primogênitos se revelaram mais ciumentos, angustiados, neuróticos e medrosos.

Adler (1929/1969; 1954/1912) sugeriu que o filho único tende a acreditar que deve ser o centro das atenções e que o seu temor maior é vir a ser destronado por um irmão mais jovem. Nas palavras do autor, "...ele realmente sente que este é seu direito e, se a sua posição é ameaçada, pensa que isto é uma grande injustiça. Na sua vida posterior, quando não é mais o centro das atenções, poderá apresentar dificuldades" (Adler, 1954/1912, p. 152). Algumas pesquisas indicaram limitações em habilidades sociais, maior incidência de comportamentos problemáticos e intervenções terapêuticas na infância de filhos únicos (Eckstein, 2000). Por outro lado, estes aspectos já foram atribuídos à monitoração excessiva e a superproteção parental (Dunn \& Plomin, 1990; Falbo, 1984). De acordo com as ideias seminais de Adler (1954/1912), o filho único é tanto o alvo das elevadas expectativas parentais, geralmente reservadas para os 
primogênitos, quanto recebe os favores e cuidados típicos de um filho caçula. Para Dunn e Plomin (1990), alguns destes indivíduos, ao tentar preencher todas as expectativas parentais, acabam por apresentar déficits de autonomia em comparação às demais posições de nascimento.

O primogênito desfruta de um período mais ou menos longo de acesso exclusivo à atenção dos pais. Após este período, ele é destronado, uma expressão que se tornou popular e que se refere à experiência do primogênito diante do nascimento de um segundo filho. No estudo de Feiring, Lewis e Jaskir (1983), a interação dos pais com os seus primogênitos dois anos mais velhos se mostrou mais vigilante, menos disposta para brincadeiras e mais tensa após o nascimento do irmão. O estresse causado por esta experiência leva a criança a sentimentos de ciúme e amargura, que podem permanecer reprimidos e resultar em insegurança na vida adulta. Embora outras crianças, tais como o caçula, possam experimentar sentimentos de destronamento, estes não serão tão intensos, pois elas já teriam vivenciado a cooperação com outros irmãos (Adler, 1954/1912). O destronamento é frequentemente citado em casos de crianças encaminhadas para intervenção psicológica, e uma alta incidência de problemas comportamentais é registrada em primogênitos destronados (Dunn \& Kendrick, 1982). Kalmuss e Davidson (1992) sugeriram que o maior flagelo dos primogênitos é a inexperiência dos pais, que experimentam maior ansiedade e são menos competentes por ocasião do primeiro filho. Diante de cuidados inconsistentes, o primogênito pode desenvolver insegurança, dependência e medos irracionais.

Vários estudos indicaram que os primeiros filhos tendem a ser mais dependentes da aprovação de outras pessoas, e, portanto, mais sugestionáveis por figuras de autoridade (Eckstein, 2000), apoiando as ideias de Adler (1954/1912), que sugeriu que primogênitos destronados tentam recuperar sua posição atendendo as expectativas dos pais. Neste sentido, Newman, Higgins e Vookles (1992) concluíram que estes indivíduos dependem mais dos padrões externos, ditados pelos pais ou outras pessoas, para pautar o seu comportamento e formar a sua autoavaliação, em comparação aos não primogênitos.

Algumas pesquisas sugerem que primogênitos e filhos únicos tendem a buscar a interação social como forma de lidar com o estresse, mais do que outras posições ordinais. Este comportamento foi atribuído a maior rapidez com que mães primíparas respondem ao chamado dos filhos, resultando na criança a expectati- va de que outros significativos podem ser imediatamente confortadores em situações difíceis. Neste sentido, pesquisadores sugeriram que primogênitos sejam mais propensos a responder à ansiedade com a afiliação (Eckstein, 2000).

Os registros de maior realização acadêmica de primogênitos (Marjoribanks, 1997) estimularam estudos que constataram que os pais tendem a ter expectativas irrealistas e a exercer pressões indevidas sobre o desempenho destas crianças em comparação com os filhos subsequentes. Isto pode instigar um desejo acentuado de realização por parte da criança, gerar ansiedade diante da possibilidade de não corresponder aos padrões parentais e baixa autoestima se chegar à conclusão que não é bom o bastante (Dunn \& Plomin, 1990; Marjoribanks, 1997). Por outro lado, os pais tendem a perceber os primogênitos como mais capazes e inteligentes, outorgando-lhes, mais frequentemente, o controle das situações. Neste sentido, os mais velhos tendem a incorporar o papel parental diante dos mais jovens. Esta postura pode despertar atitude de responsabilidade, cuidado e proteção em relação aos mais fracos; e também poderá, quando exagerada, resultar no desejo de manter os demais dependentes e ditar regras (Adler, 1954/1912; Dunn \& Plomin, 1990). De modo geral, as características típicas destes indivíduos incluem autoconfiança, perfeccionismo, habilidades de organização, metas elevadas de realização, inclinação acadêmica, conservadorismo, valorização do sucesso e boas habilidades de comunicação com os adultos (Eckstein, 2000).

Revisões das pesquisas sobre ordem de nascimento apontam como um dos resultados mais consistentes a tendência dos primogênitos para alcançarem maior nível educacional e apresentarem QI mais elevado (Zajonc, 2001). Em contraste aos estudos com homens, Bohmer e Sitton (1993) compararam a ordem de nascimento de 201 mulheres consideradas expoentes na sua área profissional e encontraram que as caçulas selecionaram carreiras científicas e se destacaram mais frequentemente do que as demais.

Os filhos 'do meio', aqueles que possuem tanto irmãos mais velhos quanto mais jovens, nunca esperam uma atenção parental completa. O estereótipo de uma criança 'do meio' negligenciada parece ter algum fundamento, que pode ser o sentimento internalizado de uma falta de papel específico no grupo familiar, especialmente após o nascimento do irmão mais jovem (Eckstein, 2000). A negociação das atenções e espaços com primogênitos potencialmente dominadores e caçulas vorazes por atenção pode desenvolver 
habilidades diplomáticas nos filhos 'do meio', assim como maior resiliência a estressores. Foi sugerido que estas crianças se tornam pessoas menos ansiosas, despreocupadas, disponíveis para o lazer e extrovertidas. E com propensão a mostrar independência, flexibilidade, diplomacia e generosidade, o que vem a contrastar com a ambição e a rigidez dos primogênitos (Eckstein, 2000; Ernst \& Angst, 1983).

As crianças mais jovens ou caçulas são supostamente mais capazes de estabelecer o seu ritmo de desenvolvimento e aprendizagem sem experienciar exaustão psicológica, pois geralmente não sofrem as pressões dispensadas aos mais velhos (Eckstein, 2000). Elas tendem a desenvolver boas habilidades sociais, promovidas pela constante interação com irmãos. Indivíduos que ocuparam esta posição têm sido descritos como seguros, mas ainda dependentes, o que é atribuído a presença de muitos cuidadores na infância. Estas pessoas podem apresentar dificuldades para estabelecer autonomia na vida adulta e sentimentos de inferioridade e de que não são levadas a sério (Richardson \& Richardson, 1990). Esta condição seria o resultado de perceber, quando crianças, que todos os demais eram maiores, mais fortes e competentes, e achar que nunca iriam competir em bases iguais e justas (Ernst \& Angst, 1983).

Na medida em que precisam competir pela atenção dos pais num ambiente em que outros já ocuparam os nichos familiares mais disponíveis, os caçulas podem se tornar mais flexíveis e abertos à mudança, procurando estratégias alternativas e mais criativas diante de situações desfavoráveis (Gould, 1997). Porém, tendo sido tratados como os 'bebês da casa', geralmente não recebem reconhecimento suficiente por suas realizações e podem vir a se rebelar ou simplesmente desistir de agradar às figuras de autoridade. Neste sentido, tendem a assumir mais riscos, a serem mais criativos, rebeldes e pouco disciplinados (Toman, 1993).

Sulloway (1999) salientou os efeitos interativos entre sexo e ordem de nascimento. A partir da revisão exaustiva de pesquisas anteriores, com destaque para os estudos pioneiros e sofisticados de Koch (e.g., Koch, 1955), posteriormente revisados por Brim (citado por Sulloway, 1999), o autor constatou que a ordem de nascimento exerce uma influência significativa no comportamento relacionado ao gênero, esclarecendo o que segue.

Uma das razões por que a ordem de nascimento interage com o gênero com tanta frequência é que essas duas variáveis promovem estratégias similares.
Os organismos sociais buscam acesso aos recursos valorizados de duas maneiras fundamentais: a dominação e a cooperação. $\mathrm{O}$ comportamento promovedor do status é uma tendência dos primogênitos e também uma tendência 'masculina'. A cooperação é uma tendência dos caçulas e também uma tendência 'feminina' (Sulloway, 1999).

A reanálise dos dados de Koch (e.g., Koch, 1955) feita por Brim (citado por Sulloway, 1999) dividiu as medidas comportamentais usadas no estudo original em traços "instrumentais" e "expressivos", para caracterizar os papéis masculino e feminino, respectivamente. As tendências masculinas incluíram liderança, competitividade, assertividade e agressão; e as femininas incluíram a afeição, a cooperação e a flexibilidade. As análises mostraram que os primogênitos de ambos os sexos destacam-se pelos traços instrumentais, e as tendências expressivas foram predominantemente manifestadas pelas meninas e pelos caçulas.

Os meninos caçulas (especialmente os que têm uma irmã mais velha) foram considerados particularmente 'efeminados' por seus professores. As meninas consideradas flexíveis e agradáveis foram julgadas ainda mais dotadas destes traços quando eram as irmãs caçulas. Nas díades que incluíam uma primogênita e um menino caçula, as meninas eram de fato mais masculinas do que os meninos (Sulloway, 1999).

Sulloway (1999) afirma que a ordem de nascimento determina muitos traços associados aos estereótipos de gênero; e sugere que "o estudo do gênero é o estudo da ordem de nascimento, e vice-versa" (p. 83). Ora, muito do comportamento relacionado ao gênero, assim como os efeitos da ordem de nascimento, é um resultado da dinâmica do sistema familiar. Sampaio (2007) argumentou que "duas variáveis em especial podem contribuir de maneira significativa para a organização familiar, o gênero e a ordem do nascimento e que, portanto, não podem ser desconsideradas" (p. 151). Porém, Sampaio (2007) alertou sobre a inconsistência dos achados das pesquisas e ressaltou a necessidade de mais estudos sobre estes temas, especialmente no Brasil.

Nos últimos dez anos, somente duas pesquisas brasileiras sobre ordem de nascimento estão registradas em bancos de dados eletrônicos (Index Psi Periódicos Técnico-Científicos; PEPsic - Periódicos Eletrônicos em Psicologia). Estes são os trabalhos de La Rosa (1998), que mostrou a importância da interação entre sexo, nível socioeconômico e ordem de nascimento para os níveis de ansiedade traço-estado. E o estudo 
de Magalhães (2008), que revelou a influência da ordem de nascimento sobre os interesses vocacionais. Sendo assim, Sampaio (2007) observou que "pesquisadores brasileiros parecem estar fora da discussão internacional que cresce na tentativa de compreender as contradições e sanar as dificuldades de avaliação dos fatores presentes nas relações familiares" (p. 151).

A construção de uma teoria, em grande parte, significa a criação de regras gerais a serem aplicadas aos casos individuais. Adler (1954/1912) descreveu tendências de comportamento associadas às diferentes posições ordinais e esclareceu que não é o número da criança na ordem de nascimentos sucessivos que influencia o seu caráter, mas a situação em que nasce e como a interpreta. A ordem de nascimento, assim como outras informações igualmente relevantes, auxilia a construção de hipóteses sobre o estilo de vida ou personalidade. Portanto, o autor esclareceu que as suas descrições devem ser encaradas como premissas gerais, um ponto de partida para a investigação da individualidade. Por exemplo, se o primogênito apresenta limitações, o segundo filho pode assumir as características de uma criança mais velha. $\mathrm{Ou}$, se numa família numerosa duas crianças nascem muito mais tarde do que as demais, e se desenvolvem juntas como uma dupla destacada do grupo, a mais velha poderá desenvolver os traços de um primogênito (Adler, 1954/1912).

Adler (1954/1912) propôs que a criança, a partir das suas experiências e observações no contexto familiar, desenvolve um plano e uma estratégia própria para inserir-se no ambiente social e alcançar status e reconhecimento. Este plano, denominado estilo de vida na terminologia adleriana, permanece relativamente estável durante a vida. As estratégias elaboradas funcionam como âncoras do estilo interpessoal do sujeito, isto é, o protótipo básico de sua maneira habitual de resolver as questões interpessoais da vida. Sulloway (1999) salientou que "o que há de sistemático na ordem de nascimento é a essência geral das estratégias, e não comportamentos específicos empregados para atingir esses fins" (p. 82). Portanto, a ambiguidade dos achados nas pesquisas sobre ordem de nascimento pode ter sido o resultado de uma ênfase em comportamentos específicos ao invés de focar as estratégias utilizadas pelos indivíduos para enfrentar os estressores particulares de suas posições ordinais e alcançar aceitação e status. Neste sentido, Sulloway (1999) sugeriu que a consistência dos efeitos da ordem de nascimento pode ser encontrada no exame do estilo de vida, isto é, na avaliação da forma particular do sujeito lidar com as situações que se apresentam. A presente pesquisa pretende contribuir nesta linha de investigação.

Em síntese, a revisão da literatura indica que a avaliação das estratégias individuais de busca de aceitação e status social pode ser útil no entendimento dos efeitos da ordem de nascimento sobre o desenvolvimento humano. Estas estratégias constituem o estilo interpessoal, uma expressão fundamental do estilo de vida, na abordagem de Adler (1954/1912). Curlette, Wheeler e Kern (1997) desenvolveram o BASIS-A (Basic Adlerian Scales of Interpersonal Styles), um conjunto de escalas para mensurar traços de estilo interpessoal dentro de uma perspectiva adleriana. E, considerando que Sulloway (1999) destacou as diferenças de estratégias interpessoais associadas ao sexo, o objetivo desta investigação foi examinar se existem efeitos combinados de sexo e ordem de nascimento sobre os traços de estilo interpessoal mensurados pelo BASIS-A. Deste modo pretende-se avançar no entendimento da essência geral das estratégias comportamentais que caracterizam diferentes posições na genitura familiar. Esta pesquisa também adquire relevância por abordar variáveis pouco investigadas no Brasil e que tem ocupado um número crescente de pesquisadores no cenário internacional (Sampaio, 2007).

\section{MÉTODO}

\section{Participantes}

Participaram do estudo 435 estudantes de ambos os sexos (196 homens e 239 mulheres), com idade entre 18 e 40 anos $(\underline{M}=23,3)$, matriculados em diversos cursos de uma universidade particular do sul do Brasil (psicologia, serviço social, administração, educação física, matemática e engenharias).

\section{Instrumentos e Procedimentos}

A pesquisa foi realizada mediante aprovação do Comitê de Ética da instituição de afiliação do pesquisador. Foram atendidas as determinações éticas da resolução 196/96 do Conselho Nacional de Saúde (CNS) sobre a pesquisa com seres humanos.

As variáveis de estilo interpessoal foram medidas através do BASIS-A (Basic Adlerian Scales of Interpersonal Styles) desenvolvido por Curlette, Wheeler e Kern (1997). Os autores, a partir das ideias de Adler (1912/1954, 1929/1969), construíram este instrumento partindo da premissa de que as percepções infantis sobre o meio social formam crenças que permanecem 
como o referencial orientador do indivíduo na sua busca pelo sucesso nas relações sociais. Portanto, o propósito do BASIS-A é identificar estas crenças e estratégias prototípicas, e compreender como afetam o comportamento social do indivíduo no presente. $\mathrm{O}$ BASIS-A é um inventário composto de 65 itens, distribuídos em 5 escalas básicas e 5 escalas suplementares, que informam sobre tendências comportamentais no contexto social. A interpretação de cada escala está baseada em evidências empíricas acumuladas em extensos estudos de validação (Curlette, Wheeler \& Kern, 1997). O cabeçalho do instrumento dita o início de todas as questões com a introdução "Quando eu era criança, eu...", que tem continuidade, em cada item, na descrição de comportamentos específicos. A versão brasileira do BASIS-A foi aplicada numa amostra de 828 estudantes universitários (Magalhães, 2005). A análise fatorial destes dados revelou uma representação adequada das escalas do instrumento, explicando $43 \%$ da variância. As escalas da versão brasileira do BASIS-A mostraram índices de consistência interna (alfa de Cronbach) entre 0,76 e 0,84 (Magalhães, 2005).

As descrições a seguir representam o significado geral das cinco escalas básicas: (a) Pertença/Interesse Social: indica extroversão e prazer de estar em grupo, capacidade de empatia e popularidade (ex: "...me enturmava facilmente num grupo"); (b) Acompanhamento: indica a preferência por situações estruturadas, aquiescência e afastamento de conflitos (ex: "...me envolvia em muitas brigas na escola" - escore invertido); (c) Comando: indica a busca de controle, dominância e liderança (ex: “...era mandão”); (d) Reconhecimento: reflete a busca por sucesso e realização de padrões de desempenho associada ao desejo por respeito e aprovação (ex: “...me sentia importante quando fazia as coisas certas"); e (e) Cautela: avalia o quanto o indivíduo percebe o seu em torno social como hostil ou desfavorável, resultando numa abordagem cuidadosa das situações que pode oscilar para o extremo oposto de falta de cuidado e imprudência; também está associada a sensibilidade aos estados subjetivos alheios (ex: “...não podia ser sincero com meus pais”).

As cinco escalas suplementares são as seguintes: (a) Severidade: identifica a pessoa que pode querer que outros a vejam como tendo passado por grandes dificuldades e traumas na infância (ex: “...um dos meus pais me achava um caso perdido"); (b) Intitulação: indica a crença de que deve ser o centro das atenções e que, por fim, poderá conseguir as coisas da sua maneira (ex: "...conseguia tudo o que eu queria”); (c)
Ser Gostado: sugere a tendência para encontrar lugar no grupo agradando e conquistando a aprovação dos demais (ex: “...queria agradar os adultos"); (d) Busca de Perfeição: caracteriza a presença de altos padrões e a sensibilidade a enganos ou erros (ex: “...era bom em cuidar de detalhes"); e (e) Suavidade: indica uma percepção otimista dos fatos, que, quando muito acentuada, pode significar o desejo de mostrar-se numa imagem favorável (ex: “...era amigável”).

A coleta de dados foi realizada em sala de aula. Os professores, oportunamente e com autorização da direção do respectivo curso, disponibilizaram $60 \mathrm{mi}-$ nutos para o procedimento. Os alunos foram convidados a participar da pesquisa e, aqueles que concordaram, assinaram o termo de consentimento livre e esclarecido.

Os sujeitos foram agrupados de acordo com a ordem de nascimento, nas seguintes categorias: primogênitos, únicos, 'do meio' e caçulas. O primogênito foi definido como a primeira criança a nascer da união dos seus pais; o caçula como a última a nascer; o 'do meio' foi aquele com irmãos mais velhos e mais jovens; filho único aquele que nasceu e viveu sem a companhia de outras crianças na mesma residência. Foram calculadas as diferenças de idade entre o sujeito e o irmão imediatamente mais velho e/ou o mais jovem. No caso de uma diferença maior do que seis anos, seguindo a sugestão da literatura (Bohmer \& Sitton, 1993), o sujeito foi excluído da amostra para evitar imprecisão na definição da posição ordinal. Indivíduos com irmãos gêmeos, adotivos ou que informaram serem provenientes de famílias reconstituídas e que conviveram, quando crianças, com irmãos não consanguíneos, também foram excluídos por este motivo. Atendendo a estes critérios, foram excluídos 34 casos. Foram examinados os efeitos combinados de sexo e ordem de nascimento num delineamento fatorial 2X4 (2 categorias de sexo e 4 categorias de ordem de nascimento), tendo, como variáveis dependentes, as escalas de estilo interpessoal.

\section{RESULTADOS}

A Tabela 1 apresenta a estatística descritiva dos escores das escalas de estilo interpessoal em cada categoria de ordem de nascimento no grupo de mulheres. Observa-se que não houve casos de mulheres filhas únicas. A Tabela 2 apresenta a estatística descritiva dos escores das escalas de estilo interpessoal em cada categoria de ordem de nascimento no grupo de homens. 
TABELA 1

Médias e Desvios Padrão dos Escores de Estilo Interpessoal e Valores de N em Cada Ordem de Nascimento em Mulheres

\begin{tabular}{|c|c|c|c|c|c|c|c|c|c|c|c|c|}
\hline \multirow{3}{*}{$\begin{array}{l}\text { Escalas de Estilo } \\
\text { Interpessoal }\end{array}$} & \multicolumn{12}{|c|}{ Posição na ordem de nascimento } \\
\hline & \multicolumn{3}{|c|}{ Primogênito } & \multicolumn{3}{|c|}{ ‘Do meio' } & \multicolumn{3}{|c|}{ Caçula } & \multicolumn{3}{|c|}{ Total } \\
\hline & $\mathrm{N}$ & M & DP & $\mathrm{N}$ & M & $\mathrm{DP}$ & $\mathrm{N}$ & M & DP & $\mathrm{N}$ & $\mathrm{M}$ & DP \\
\hline Pertencimento & 59 & 34,22 & 5,58 & 98 & 34,34 & 7,12 & 58 & 35,71 & 5,71 & 215 & 34,67 & 6,37 \\
\hline Acompanhamento & 59 & 31,05 & 5,97 & 98 & 30,86 & 5,96 & 58 & 33,29 & 5,19 & 215 & 31,57 & 5,84 \\
\hline Comando & 59 & 21,39 & 7,54 & 98 & 20,01 & 6,70 & 58 & 19,67 & 7,95 & 215 & 20,30 & 7,28 \\
\hline Reconhecimento & 59 & 41,51 & 6,73 & 98 & 43,80 & 7,63 & 58 & 44,69 & 7,11 & 215 & 43,41 & 7,32 \\
\hline Cautela & 59 & 15,73 & 5,69 & 98 & 15,60 & 7,29 & 58 & 13,38 & 6,69 & 215 & 15,04 & 6,77 \\
\hline Suavidade & 59 & 12,55 & 3,06 & 98 & 12,68 & 3,38 & 58 & 11,78 & 2,86 & 215 & 12,40 & 3,17 \\
\hline Ser Gostado & 59 & 22,08 & 4,68 & 98 & 23,26 & 4,85 & 58 & 24,52 & 4,83 & 215 & 23,27 & 4,86 \\
\hline Perfeccionismo & 59 & 18,88 & 3,05 & 98 & 19,11 & 3,09 & 58 & 18,98 & 2,82 & 215 & 19,01 & 3,00 \\
\hline Intitulação & 59 & 18,02 & 5,67 & 98 & 16,77 & 5,17 & 58 & 17,72 & 5,79 & 215 & 17,37 & 5,48 \\
\hline Severidade & 59 & 12,76 & 2,80 & 98 & 12,90 & 2,60 & 58 & 12,78 & 3,46 & 215 & 12,83 & 2,89 \\
\hline
\end{tabular}

TABELA 2

Médias e Desvios Padrão dos Escores de Estilo Interpessoal e Valores de N em Cada Ordem de Nascimento em Homens

\begin{tabular}{|c|c|c|c|c|c|c|c|c|c|c|c|c|c|c|c|}
\hline \multirow{3}{*}{$\begin{array}{l}\text { Escalas de Estilo } \\
\text { Interpessoal }\end{array}$} & \multicolumn{15}{|c|}{ Posição na ordem de nascimento } \\
\hline & \multicolumn{3}{|c|}{ Filho único } & \multicolumn{3}{|c|}{ Primogênito } & \multicolumn{3}{|c|}{ 'Do meio' } & \multicolumn{3}{|c|}{ Caçula } & \multicolumn{3}{|c|}{ Total } \\
\hline & $\mathrm{N}$ & M & $\mathrm{DP}$ & $\mathrm{N}$ & M & DP & $\mathrm{N}$ & M & DP & $\mathrm{N}$ & M & DP & $\mathrm{N}$ & M & DP \\
\hline Pertencimento & 12 & 34,2 & 5,98 & 61 & 34,5 & 6,41 & 63 & 36,7 & 5,11 & 50 & 34,5 & 6,44 & 186 & 35,2 & 6,03 \\
\hline Acompanhamento & 12 & 28,8 & 6,88 & 61 & 30,9 & 6,02 & 63 & 31,2 & 4,79 & 50 & 30,3 & 6,79 & 186 & 30,7 & 5,90 \\
\hline Comando & 12 & 20,3 & 6,54 & 61 & 18,9 & 5,66 & 63 & 17,9 & 6,35 & 50 & 18,4 & 5,46 & 186 & 18,5 & 5,89 \\
\hline Reconhecimento & 12 & 43,1 & 5,85 & 61 & 42,5 & 6,65 & 63 & 40,8 & 7,02 & 50 & 39,2 & 7,72 & 186 & 41,1 & 7,12 \\
\hline Cautela & 12 & 13,2 & 4,95 & 61 & 14,5 & 5,45 & 63 & 12,1 & 3,55 & 50 & 13,1 & 5,02 & 186 & 13,2 & 4,79 \\
\hline Suavidade & 12 & 11,9 & 2,77 & 61 & 12,4 & 2,90 & 63 & 11,9 & 2,52 & 50 & 11,9 & 2,68 & 186 & 12,1 & 2,69 \\
\hline Ser Gostado & 12 & 22,7 & 3,79 & 61 & 23,0 & 4,29 & 63 & 21,9 & 4,88 & 50 & 20,9 & 4,81 & 186 & 22,0 & 4,65 \\
\hline Perfeccionismo & 12 & 21,1 & 2,44 & 61 & 19,5 & 3,20 & 63 & 19,1 & 2,79 & 50 & 18,7 & 3,21 & 186 & 19,2 & 3,06 \\
\hline Intitulação & 12 & 19,7 & 5,10 & 61 & 16,0 & 4,34 & 63 & 15,7 & 4,51 & 50 & 15,8 & 4,23 & 186 & 16,1 & 4,49 \\
\hline Severidade & 12 & 14,4 & 2,77 & 61 & 13,8 & 2,83 & 63 & 13,9 & 2,88 & 50 & 12,6 & 2,60 & 186 & 13,5 & 2,83 \\
\hline
\end{tabular}

A ANOVA fatorial 2X4 (2 categorias de sexo e 4 categorias de ordem de nascimento) revelou interação entre sexo e ordem de nascimento para variações em Reconhecimento [F $(2,401=6,13, \underline{\mathrm{p}}<.01)]$, Ser gostado $[\underline{\mathrm{F}}(2,401=6,53, \mathrm{p}<.01)]$, Acompanhamento $[\underline{\mathrm{F}}(2,401=3,43, \underline{\mathrm{p}}<.05)]$ e Cautela $[\underline{\mathrm{F}}(2,401$ $=4,13, \underline{p}<.05)]$. As análises de variância e testes post hoc (Scheffe, Tukey) revelaram os resultados descritos a seguir.

Mulheres caçulas mostraram escores mais elevados do que homens caçulas nas escalas Reconhecimento $[\underline{F}(1,108=14,66, \underline{p}<.01)]$, Ser Gostado [ $\underline{F}(1,108=$ $14,91, \mathrm{p}<.01)]$ e Acompanhamento $[\underline{\mathrm{F}}(1,108=6,53$, $\mathrm{p}<.05)]$. Além disto, obtiveram escores mais eleva- dos na escala de Acompanhamento em comparação com homens primogênitos $[\underline{\mathrm{F}}(1,118=5,00, \underline{\mathrm{p}}<.05)]$ e 'do meio' $[\underline{F}(1,120=4,87, \underline{p}<.05)]$.

Mulheres filhas 'do meio' mostraram escores mais elevados de Reconhecimento $[\underline{F}(1,160=6,75$, $\underline{\mathrm{p}}<.05)$ ], Ser gostado [ $\underline{\mathrm{F}}(1,160=6,05, \underline{\mathrm{p}}<.05)] \mathrm{e}$ Cautela $[\underline{F}(1,160=12,15, \underline{p}<.05)]$ em comparação com homens filhos 'do meio'.

As comparações (ANOVA) no grupo dos homens revelaram nos caçulas escores mais baixos em comparação com primogênitos e filhos únicos nas escalas Reconhecimento [ $\underline{\mathrm{F}}(3,185=2,74, \underline{\mathrm{p}}<.05)]$ e Ser gostado $[\underline{F}(3,185=2,85, \underline{p}<.05)]$. Ainda no grupo dos homens, filhos únicos revelaram escores mais 
elevados de Intitulação do que as demais categorias de ordem de nascimento [ $\underline{\mathrm{F}}(3,185=2,93, \mathrm{p}<.05)]$, e Perfeccionismo mais elevado do que os caçulas $[\underline{\mathrm{F}}(3$, $185=2,71, \mathrm{p}<.05)]$. E os primogênitos mostraram escores mais elevados em Cautela do que filhos 'do meio' $[\underline{\mathrm{F}}(3,185=2,82, \underline{\mathrm{p}}<.05)]$.

As comparações (ANOVA) no grupo das mulheres mostraram que as caçulas tem escores mais elevados do que as primogênitas em Reconhecimento [ $\underline{F}$ (2, $214=3,40, \underline{p}<.05)]$ e Ser Gostado $[\underline{F}(2,214=3,75$, $\mathrm{p}<.05)]$.

\section{DISCUSSÃO}

As mulheres caçulas mostraram escores elevados em Reconhecimento e Ser Gostado em comparação com as primogênitas e com homens caçulas. Além disto, também obtiveram escores mais elevados de Acompanhamento em comparação com homens primogênitos e 'do meio'. A combinação de escores elevados em Reconhecimento, Ser gostado e Acompanhamento sugere tendência a evitar conflitos, aquiescência, receptividade e tentativa de atender a padrões de desempenho, configurando uma estratégia geral de aceitação ou mesmo submissão às expectativas alheias. Sabendo-se que a dominação e a cooperação são as duas maneiras fundamentais dos organismos sociais buscarem acesso aos recursos valorizados (Sulloway, 1999), a estratégia predominante das mulheres caçulas parece ser a cooperação. Neste sentido, Sulloway (1999) descreveu as características dos caçulas como marcadas pelo traço de conciliatividade/amabilidade. Além disto, as mulheres caçulas podem, devido à socialização diferencial ligada ao gênero, usar mais de comportamento carismático e sedutor para obter influência no grupo. Nos estudos de Koch (citados por Sulloway, 1999) os traços expressivos de personalidade associados ao gênero feminino, foram atribuídos com mais intensidade às meninas caçulas, que foram descritas como flexíveis e agradáveis. Deste modo, os dados sugerem que mulheres caçulas prefiram exercer influência por meios não confrontadores e pela habilidade para agradar os demais.

Por outro lado, os escores elevados em Reconhecimento nas mulheres caçulas podem revelar uma busca pelo sucesso mais acentuada do que as primogênitas. Neste sentido, na amostra estudada por Bohmer e Sitton (1993), as caçulas obtiveram maior status profissional do que as primogênitas. As caçulas podem estar revelando um aspecto de rebeldia, de modo consistente com a mesma tendência descrita em homens caçulas (Sulloway, 1999), na medida em que a luta por posições de status é um comportamento ligado ao estereótipo de gênero masculino. Deste modo, estariam apresentando um comportamento não tradicional em termos dos estereótipos de gênero. Porém, no caso das mulheres, esta parece ser uma rebeldia menos ruidosa do que a masculina, considerando o imperativo de serem gostadas e não se confrontarem abertamente com as normas do grupo. Por outro lado, as primogênitas, tipicamente adeptas a agradar os adultos e agir de maneira socialmente convencional, talvez acabem por ceder à socialização feminina tradicional, que não enfatiza a ambição e o elevado sucesso profissional.

No grupo dos homens, os caçulas se mostraram com escores mais baixos em Reconhecimento e Ser gostado em comparação com primogênitos e também em relação às mulheres caçulas. Estes dados indicam uma tendência a se preocupar menos com a aprovação externa e a apresentar comportamentos menos convencionais, o que corresponde ao argumento de Sulloway (1999) sobre a "vocação rebelde" destes indivíduos (p. 13). O autor mostrou evidências de que os caçulas, ao longo da história, foram mais receptivos às inovações científicas; e os descreveu como mais abertos à experiência, argumento recentemente corroborado por Healey e Ellis (2007). Estes dados sugerem que o sexo do caçula tem influência importante no tipo de estratégia a ser utilizada na busca de um lugar no grupo familiar. Enquanto homens mostraram menor preocupação em agradar e corresponder a expectativas, as caçulas revelaram uma busca de aprovação e tendência a evitar conflitos. Uma possibilidade de entendimento dos dados é que a rebeldia atribuída aos caçulas apareça, no caso dos homens, pela recusa às ambições convencionais e pela procura de meios alternativos de reconhecimento; e, no caso das mulheres, na competição por status, um comportamento associado ao gênero masculino.

Os homens primogênitos mostraram escores elevados em Reconhecimento e Ser gostado em comparação com homens caçulas. Este dado confirma a necessidade elevada nestes indivíduos de atender às convenções, agradar e obter sucesso (Eckstein, 2000), características do traço de escrupulosidade atribuído por Sulloway (1999) a estes sujeitos. Por outro lado, os mesmos primogênitos mostraram escores mais baixos em Acompanhamento em comparação com mulheres caçulas, revelando maior capacidade para agir com independência e para entrar em conflito, corroborando uma tendência de comportamento ligada ao estereótipo masculino e, de acordo com pesquisas anteriores, predominante nos homens (Sulloway, 1999). Neste sentido, a busca por status e reconhecimento de homens primogênitos provavelmente se dá de forma 
mais assertiva e menos aquiescente do que as mulheres caçulas. A busca por reconhecimento em homens primogênitos é consistente com as inúmeras pesquisas que constataram a maior motivação para o sucesso e status em primogênitos homens, revelada na tendência para alcançarem maior nível educacional (Zajonc, 2001).

Assim como os primogênitos, filhos únicos também mostraram escores mais elevados em Reconhecimento e Ser gostado em comparação com os caçulas. Ora, estas crianças tendem a receber cuidados especiais e a ser o alvo de expectativas parentais mais elevadas quanto à realização e status profissional (Leong, Hartung, Goh \& Gaylor, 2001). Na medida em que outras crianças nascem, os pais tendem a ser mais abertos e liberais, e isto pode permitir que as mais jovens assumam maiores riscos ou escolham ocupações de menor prestígio social. Por exemplo, se o primogênito ou filho único decide ser um poeta, isto pode preocupar os pais. Mas se o caçula o faz, eles talvez não se importem tanto, uma vez que o primogênito tenha correspondido às suas expectativas de status social (Leong \& cols., 2001).

Mulheres filhas 'do meio' mostraram escores mais elevados em Reconhecimento, Ser gostado e Cautela em comparação com homens 'do meio'. A combinação dos escores elevados nestas escalas sugere uma estratégia de cooperação marcada pela sensibilidade às necessidades alheias, atendendo expectativas $\mathrm{e}$ mostrando competência. De um modo geral, crianças 'do meio' foram caracterizadas como 'espremidas' entre irmãos mais velhos e mais jovens, e com dificuldades para encontrar o seu lugar na família. Este resultado sugere para as mulheres que as habilidades diplomáticas atribuídas a esta posição (Adler, 1954/ 1912; 1969/1929) estão associadas não somente com a preocupação com serem gostadas e reconhecidas, mas também a uma sensibilidade aos estados subjetivos alheios, sugerida pelos escores elevados tanto em Cautela quanto em Ser gostado. Considerando que a cooperação é uma tendência associada ao estereótipo de gênero feminino, este resultado sugere que as mulheres reajam à condição de filhas 'do meio' com atitudes ainda mais acentuadas de busca de aceitação, docilidade e flexibilidade. Além disto, percebem esta situação como mais desfavorável do que os homens, como revelam os escores em Cautela.

Os sentimentos de intitulação e as exigências de atenção exclusiva são traços comportamentais frequentemente usados para descrever filhos únicos. Homens filhos únicos revelaram maior intitulação do que homens primogênitos, caçulas e 'do meio', o que parece confirmar este estereótipo. Ora, os filhos úni- cos recebem os favores e cuidados típicos de um filho caçula e não sofrem comparações ou dividem as atenções com irmãos mais velhos. Noutra perspectiva, a intitulação pode estar associada a crenças otimistas sobre a vida e ao sentimento de que os acontecimentos terão um desfecho favorável. Porém, esta expectativa, quando exacerbada, pode resultar em dificuldades para enfrentar as frustrações da vida de forma positiva e autônoma, ocorrendo maior necessidade de afiliação sob estresse, tal como foi sugerido por Hoyt e Raven (1973) e apontado na revisão da literatura feita por Eckstein (2000). A amostra não contou com mulheres filhas únicas, portanto, não há resultados relativos a este grupo.

Os escores elevados dos primogênitos na escala Cautela em comparação aos filhos 'do meio' sugerem que os primeiros percebem seu ambiente como mais desfavorável, no qual, portanto, devem ser cuidadosos e prevenidos. Este resultado é coerente com a descrição dos primogênitos como mais suscetíveis a sentimentos de insegurança e medo (Eckstein, 2000), apresentando características de neuroticismo (Sulloway, 1999) e, portanto, sendo mais frequentemente encaminhados para serviços de atenção psicológica (Dunn \& Kendrick, 1982; Dunn, \& Plomin, 1990). O impacto do destronamento pode resultar numa postura mais cautelosa em relacionamentos de intimidade, produzindo maior sensibilidade com relação aos estados subjetivos alheios. Além disto, no primogênito, as pressões para realizar sonhos familiares podem gerar ansiedade diante da possibilidade de não corresponder aos padrões parentais e baixa autoestima se chegar à conclusão de que não é bom o bastante (Adler, 1912/ 1954; Thomas \& Marchand, 1993).

Em síntese, observa-se que, de modo particular em homens e mulheres, as diferenças encontradas entre as categorias de ordem de nascimento corroboraram descrições da literatura. Nos homens, a "vocação rebelde" dos caçulas (Sullivan, 1999, p. 13), a cautela e a insegurança dos primogênitos, associadas ao desejo de atender a expectativas externas (Eckstein, 2000; Newman, Higgins \& Vookles, 1992), e a intitulação dos filhos únicos (Adler, 1929/1969) foram representados nos traços de estilo interpessoal do BASIS-A. Porém, estas diferenças não foram evidenciadas nas mulheres. Mulheres caçulas não se mostraram menos desejosas de reconhecimento e de aprovação social, tal como ocorreu com os homens caçulas. O maior desejo de reconhecimento e a postura mais cautelosa dos primogênitos homens não foram evidenciados no grupo das mulheres. Por outro lado, mulheres 'do meio' e caçulas revelaram as inclinações mais acentuadas para a amabilidade e a aquiescência interpessoal 
apontadas na revisão da literatura (Sulloway, 1999). Mulheres 'do meio' também mostraram uma percepção mais desfavorável do seu ambiente social, de modo coerente às dificuldades teoricamente associadas a sua posição na genitura familiar (Thomas \& Marchand, 1993). Estes resultados sugerem que a socialização diferenciada dos sexos em nossa cultura tenha impacto sobre o modo como cada posição de nascimento é experimentada. Portanto, indicam que o exame da combinação entre sexo e ordem de nascimento contribui para o entendimento do repertório das estratégias que cada indivíduo desenvolve no esforço de ocupar um lugar significativo no seu ambiente social.

Neste sentido, em termos de aplicações práticas, os resultados apontam a ordem de nascimento como uma informação valiosa para o entendimento de queixas apresentadas por indivíduos em psicoterapia. Neste contexto de aplicação, agrega uma perspectiva de compreensão do significado dos comportamentos adultos, considerados estratégias de ajustamento ao ambiente familiar da infância que permaneceram ativas como um estilo pessoal de conquistar aceitação e reconhecimento social ao longo da vida.

Ainda no âmbito da clínica psicológica, sabe-se que a ordem de nascimento é um aspecto relevante em processos de avaliação e intervenção em sistemas familiares. Os resultados deste estudo reforçam conclusões anteriormente apresentadas por profissionais desta especialidade (Christensen, 1993; McGoldrick, Gerson \& Shellenberger, 1999). McGoldrick, Gerson e Shellenberger (1999) consideram que o conhecimento da ordem de nascimento pode trazer explicações normativas úteis sobre os papéis dos indivíduos em suas famílias, assim como indicam outros fatores a serem explorados quando os padrões comportamentais típicos não são encontrados.

O conhecimento das implicações psicológicas da ordem de nascimento também pode ser utilizado em programas de educação parental. A educação ou o aconselhamento parental é uma prática comum em países da Europa e da América do Norte, sendo exercida por profissionais da saúde e da educação. Esta atividade tem por objetivo preparar e orientar pais para se tornarem agentes conscientes e eficazes do desenvolvimento psicossocial dos seus filhos. Acredita-se que os conceitos de diversificação evolutiva, nichos familiares e ordem de nascimento podem ser úteis neste sentido. A ordem de nascimento aparece como um fator relevante no programa de educação parental STEP (Systematic Training for Effective Parenting) desenvolvido por Dinkmeyer, Mckay e
Dinkmeyer (1997), um dos mais difundidos internacionalmente.

Por fim, os resultados deste estudo reforçam as ideias dos defensores da ordem de nascimento como uma variável importante na explicação de diferenças de personalidade (Adler, 1929/1969; Sulloway, 1999), e corroboram as tendências encontradas na literatura. Além disto, sugerem que há uma relação significativa entre sexo e ordem de nascimento na definição das estratégias que o indivíduo utiliza para se inserir no espaço social. Para dar continuidade a esta linha de investigação, recomendam-se estudos mais rigorosos que investiguem os efeitos da ordem de nascimento através de comparações entre indivíduos da mesma família e considerem a composição sexual do grupo de irmãos. Outros aspectos a serem examinados em associação a ordem de nascimento são as percepções sobre práticas educativas parentais e o impacto do nível socioeconômico.

\section{REFERÊNCIAS}

Adler, A. (1954). El carácter neurótico. Buenos Aires: Paidós.

Adler, A. (1969). The science of living. Nova York: Anchor Books.

Beer, J. M., \& Horn, J. M. (2000). The influence of rearing order on personality development within two adoption cohorts. Journal of Personality, 68, 789-819.

Belmont, L. (1977). Birth order, intellectual competence and psychiatric status. Journal of Individual Psychology, 33, 97-104.

Bohmer, P., \& Sitton, S. (1993). The influence of birth order and family size on notable american women's selection of careers. Psychological Record, 43(3), 124-132.

Brim, O. G., Jr. (1958). Family structure and sex-role learning by children: A further analysis of Helen Koch's data. Sociometry, 21, 1-16.

Christensen, O. C. (Org.) (1993). Adlerian family counseling. Minneapolis: EMC.

Curlette, W. L., Wheller, M. S., \& Kern, R. M. (1997). Basis-A Inventory Technical Manual. Highlands, NC: TRT Associates.

Dinkmeyer, D., Mckay, G. D., \& Dinkmeyer, D. (1997). The parent's handbook. Circle Pines, MN: American Guidance Service.

Dreikurs, R. (1963). Individual psychology: The Adlerian point of view. Em J. M. Wepman \& R. Heine (Orgs.), Concepts of personality (pp. 147-162). Chicago: Aldine.

Dunn, J., \& Kendrick, C. (1982). Siblings. London: Grant McIntyre.

Dunn, J., \& Plomin, R. (1990). Separate lives: Why siblings are so different. New York: Basic Books.

Eckstein, D. (2000). Empirical studies indicating significant birth-order related personality differences. Journal of Individual Psychology, 56(4), 481-494. 
Elliott, B. A. (1992). Birth order and health: Major issues. Social Science \& Medicine, 35, 443-452.

Ernst, C., \& Angst, J. (1983). Birth order. Berlin: SpringerVerlag.

Falbo, T. (1984). The single-child family. New York: Guilford Press.

Feiring, C., Lewis, M., \& Jaskir, J. (1983). Birth of a sibling: Effect on mother-first born child interaction. Journal of Developmental Behavioral Pediatrics, 4, 190-195.

Gould, S. J. (1997). Dolly's fashion and Louis's passion. Natural History, 106, 18-24.

Healey, M. D., \& Ellis, B. J. (2007). Birth order, conscientiousness, and openness to experience: Tests of the familyniche model of personality using a within-family methodology. Evolution and Human Behavior, 28, 55-59.

Hoyt, M. P., \& Raven, B. H. (1973). Birth order and the 1971 earthquake. Journal of Personality and Social Psychology, 20, 122-128.

Kalmuss, D., \& Davidson, A. (1992). Parenting expectations, experiences, and adjustment to parenthood: A test of the violated expectations framework. Journal of Marriage \& the Family, 54, 516-526.

Koch, H. L. (1955). Some personality correlates of sex, sibling position, and sex of sibling among five- and sixyear-old children. Genetic Psychology Monographs, 52, 3-50.

La Rosa, J. (1998). Ansiedade, sexo, nível socioeconômico e ordem de nascimento. Psicologia: Reflexão e Crítica, 11(1), 59-70.

Leong, F. T., Hartung, P. J., Goh, D., \& Gaylor, M. (2001). Appraising birth order in career assessment: Linkages to Holland's and Super's models. Journal of Career Assessment, 9, 25-39.

Magalhães, M. (2005). Adaptação e validação da versão brasileira das Escalas de Estilos Interpessoais (BASISA). Em Sociedade Brasileira de Psicologia (Org.), Resumos da XXXV Reunião Anual de Psicologia [CD-ROM]. Curitiba, PR: SBP.

Magalhães, M. O. (2008). Relação entre ordem de nascimento e interesses vocacionais. Estudos de Psicologia, 5(2), 203-210.

Marjoribanks, K. (1997). Ordinal position, family environment, and status attainment among Australian young adults. The Journal of Social Psychology, 137, 398-399.
McGoldrick, M., Gerson, R., \& Shellenberger, S. (1999). Genograms: Assessment and intervention. New York: W. W. Norton.

Newman, L. S., Higgins, E. T., \& Vookles, J. (1992). Selfguide strength and emotional vulnerability: Birth order as a moderator of self-affect relations. Personality and Social Psychology Bulletin, 18, 402-411.

Richardson, R. W., \& Richardson, L. A. (1990). Birth order and you. North Vancouver: Self-Counsel Press.

Sampaio, I. T. A. (2007). Práticas educativas parentais, gênero e ordem de nascimento dos filhos: Atualização. Revista Brasileira de Crescimento e Desenvolvimento Humano, 17(2), 144-152.

Saroglou, V., \& Fiasse, L. (2003). Birth order, personality, and religion: A study among young adults from a threesibling family. Personality and Individual Differences, 35, 19-29.

Sulloway, F. J. (1999). Vocação rebelde: Ordem de nascimento, dinâmica familiar e vidas criativas. São Paulo: Record.

Sulloway, F. J. (2001). Birth order, sibling competition and human behavior. Em H. R. Holcomb, III (Org.), Conceptual challenges in evolutionary psychology: Innovative research strategies (pp. 39-84). Boston: Kluwer.

Thomas, C. R. \& Marchand, W. C. (1993). Basic principles of adlerian family counseling. Em O. C. Christensen (Org.), Adlerian family counseling (pp. 7-26). Minneapolis: EMC.

Toman, W. (1993). Family constellation. New York: Springer.

Zajonc, R. B., \& Mullally, P. R. (1997). Birth order: Reconciling conflicting effects. American Psychologist, 52, 685-699.

Zajonc, R. B. (2001). The family dynamics of intellectual development. American Psychologist, 56(6/7), 490-496.

Zweigenhaft, R. (2002). Birth order effects and rebelliousness: Political activism and involvement with marijuana. Political Psychology, 23, 219-233.

Recebido: 24/10/2007

Última revisão: 23/12/2008

Aceite final: 12/02/2009

\section{Sobre o autor:}

Mauro de Oliveira Magalhães: Doutor em Psicologia (UFRGS), Professor da Universidade Luterana do Brasil (ULBRA - Canoas, RS), Pesquisador visitante no Programa de Pós-graduação em Psicologia da Universidade Federal da Bahia (UFBA).

Endereço para correspondência: Rua Tenente Pires Ferreira, 308 - Bloco A - Edifício Vendaval, apto. 201 - 40130-160 Salvador - BA Endereço eletrônico: mauro.m@terra.com.br. 